\title{
How to Effectively Use Multiple Channels in Wireless Mesh Networks
}

\author{
Pan Li, Member, IEEE, Nicola Scalabrino, Yuguang (Michael) Fang, Fellow, IEEE, \\ Enrico Gregori, and Imrich Chlamtac, Fellow, IEEE
}

\begin{abstract}
Operating on a frequency band occupying several nonoverlapping channels, IEEE 802.11 is now widely used in Wireless Mesh Networks (WMNs). Many multichannel MAC protocols are proposed to improve the spatial reuse in the network under the assumption that the transmissions on nonoverlapping channels do not interfere with each other. Some joint routing and channel assignment algorithms are also designed to increase the network throughput based on the premise that we can switch between different channels freely. Although simulations show that great improvements on network throughput can be observed in both cases, two fundamental questions remain: 1) Can we really use multiple nonoverlapping channels freely in WMNs? 2) If we can, what will be the cost when we switch channels dynamically and frequently? In this paper, by conducting extensive experiments on our testbed, we attempt to answer these questions. We find that in spite of interference between both overlapping and nonoverlapping channels, we can still use multiple channels in mesh networks under certain conditions but with care. We also show that the channel switching cost is actually very significant in WMNs. We recommend not to switch the channels too frequently when designing the channel assignment algorithms, and those channel assignment algorithms selecting one channel for each packet are not really beneficial.
\end{abstract}

Index Terms-Multiple channels, channel interference, channel switching cost, wireless mesh networks.

\section{INTRODUCTION}

$\mathrm{N}$ OWADAYS, wireless networks have gained popularity and are widely used in people's daily life. For example, many people install wireless routers in their houses or offices to provide the last-mile access network. By doing this, people can walk around carrying their mobile devices and get access to the Internet freely. It is very convenient since no wired connection is needed. However, this unplanned free use of wireless resource may lead to significantly degraded network performance due to the serious interference from the neighborhood. As a result, we need a well-planned wireless network that can provide Internet access to all the users in an area, while maintaining good performance. Wireless Mesh Network (WMN) is such a solution.

A typical WMN architecture is shown in Fig. 1, where the access points (APs) (or mesh routers) are rarely mobile and may not have power constraints. In addition, these networks behave almost like wired networks in having infrequent topology changes, limited node failures, etc. In centralized WMNs [8], some mesh routers are also

- P. Li is with the Department of Electrical and Computer Engineering, Mississippi State University, Starkville, MS 39762.

E-mail: li@ece.msstate.edu.

- N. Scalabrino is with Thales Security Solutions \& Services Division, 50019, Sesto Fiorentino (FI), Italy.

E-mail: nicola.scalabrino@thalesgroup.com.

- Y. Fang is with the Department of Electrical and Computer Engineering, University of Florida, Gainesville, FL 32611. E-mail: fang@ece.ufl.edu.

- E. Gregori is with the Institute of Informatics and Telematics, Italian National Research Council (CNR), 56124, Pisa, Italy.

E-mail: enrico.gregori@iit.cnr.it.

- I. Chlamtac is with CREATE-NET, Via dei Solteri 38, 38100 Trento, Italy. E-mail: imrich.chlamtac@create-net.org.

Manuscript received 1 July 2008; accepted 2 Dec. 2008; published online 10 Dec. 2008.

Recommended for acceptance by P. Mohapatra.

For information on obtaining reprints of this article, please send e-mail to tpds@computer.org, and reference IEEECS Log Number TPDS-2008-07-0248. Digital Object Identifier no. 10.1109/TPDS.2008.256.

$1045-9219 / 09 / \$ 25.00$ C 2009 IEEE equipped with a gateway capability through which they connect to the wired network. In such networks, traffic is mainly routed by the WMN wireless backbone nodes (BNs) between the mesh clients and the Internet, to and from the gateway nodes.

Although initially standardized for Wireless Local Area Networks (WLANs), IEEE 802.11 standard is now also used for WMNs and has become the de facto. Since the standard defines multiple channels, many researchers propose to utilize multiple channels to improve the network throughput. Especially, some multichannel MAC protocols like [15], [19], and [29], and some joint routing and channel assignment algorithms like [25] and [26] are developed to reduce the interference in the network. However, these protocols and algorithms may not work efficiently for two reasons. First, those multichannel MAC protocols assume that there is no interference between two nonoverlapping channels. But it has been shown in [21], [22], and [28] that this is not true. Second, many joint routing and channel assignment algorithms utilize multiple radios and multiple channels at each node. Each radio changes from one channel to another after some time, depending on their specific schemes. Unfortunately, those algorithms do not take the channel switching cost into consideration. How channel switching affects the network performance is still unknown.

Thus, there arise two questions:

Q1. Can we really use multiple channels freely in WMNs?

Q2. If we can, what will be the cost when we switch the channel dynamically and frequently?

In this paper, we attempt to answer these two questions by conducting extensive experiments on our indoor wireless mesh testbed and hence provide some guidelines for using multiple channels in WMNs. We first show that although there is interference between two nonoverlapping channels (and also two overlapping channels), we can still 


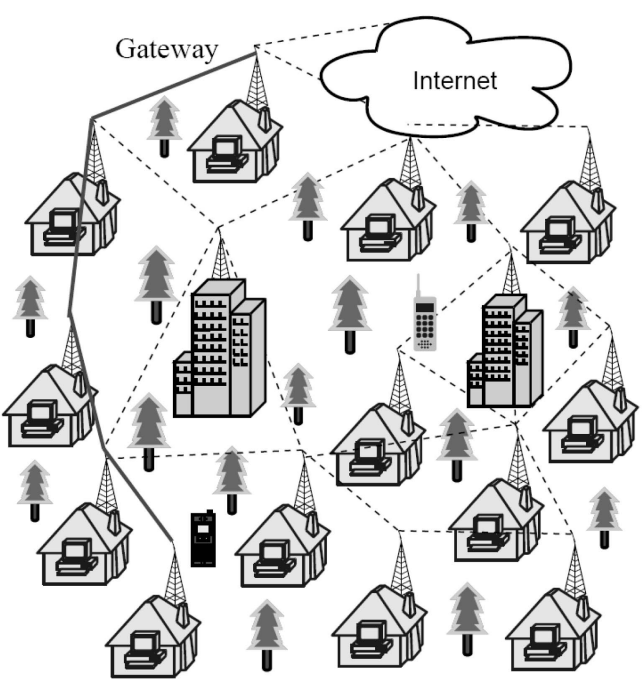

Fig. 1. Typical mesh architecture. The bold line represents the four-hop path under consideration.

use multiple channels in WMNs without degraded network performance if either the traffic in the neighborhood of each receiver is unsaturated or the radios using different channels are placed far apart from each other. We quantify the distances required for possible interfering transmitters not to interfere with the receiver under consideration. A lower bound on the minimum distance required for each possible interfering transmitter not to interfere with the receiver under analysis is given.

We also show that the channel switching cost is actually very significant. For a chain topology with four hops, the channel switching time, which is the time for the network to reach the steady state again after switching the channel, is around 10 seconds using Linksys WRT54GL wireless routers and optimized link-state routing (OLSR). Using static routing can reduce this channel switching time to around 6 seconds, which is still high. We also carry out some experiments using another kind of widely used wireless router, i.e., Netgear WGT634U routers. Experimental results show that there is no big difference between these two kinds of hardware. The channel switching time is on the time scale of several seconds in both cases. Thus, we suggest that we should not switch the channels too frequently when designing the channel assignment algorithms. Especially, those channel assignment algorithms selecting one channel for each packet are inappropriate.

The rest of this paper is organized as follows: Related work is shown in Section 2. In Section 3, we present the configuration of our testbed. We conduct some experiments in Section 4 to study the impacts of interchannel interference on network performance, i.e., the question Q1. More experimental results are given in Section 5 to show the cost of switching channels in a mesh network, i.e., the question Q2. In Section 6, we study the dependency of channel switching cost on routing protocols and hardware. Finally, we conclude this paper in Section 7.

\section{Related Work}

In the literature, there are many papers on designing MAC protocols and channel assignment algorithms for wireless networks using multiple channels, such as [9], [25], [29], and [31]. They show that the network performance can be improved significantly compared to the conventional wireless networks using a single channel. However, the results in [9], [29], and [31] are based on simulations where they do not consider the cost of channel switching, and the results in [25] are based on a testbed composed of PCs with wireless cards, which is much different from our case because the operating systems (OSs) and the abilities to process data are different.

References [21], [22], and [28] study the interchannel interference problem and show that there does exist interference between nonoverlapping channels. Mishra et al. quantify in [23] and [24] the minimum distance required for two radios operating on two different channels not to interfere with each other. However, they assume that transmitters and receivers use the same signal filter, and they only use the free space power propagation model to predict the interference introduced by a transmitter at one receiver on another channel, which is not so practical. In this paper, we estimate the interference by assuming different signal filters for receivers ${ }^{1}$ and using a general power propagation model. Multiple, instead of single (as that in [24]), interfering nodes are considered here. We find that even though there is some interference between different channels, we can still use multiple channels in a network without degraded performance if either the traffic in the neighborhood of each receiver is unsaturated or the distances from interfering nodes to the receiver under consideration satisfy certain conditions.

Bahl et al. [11] propose a channel hopping protocol that can utilize multiple channels to increase the throughput in IEEE 802.11 ad hoc networks. They study the impacts of channel switching on routing protocols. Specifically, the neighbors using a multichannel MAC on different channels could cause broadcasts to reach significantly fewer neighbors than for a single-channel MAC. Thus, a broadcast retransmission scheme is introduced to address this problem. However, in this paper, we study a very different problem. We try to find the channel switching time, i.e., the time for the network to reach the steady state again after switching the channel, the end-to-end delay, and the packet loss ratio when switching channels.

Herzel et al. [17] point out that the device-level channel switching latency in an IEEE 802.11 Network Interface Card (NIC) is approximately $80 \mu \mathrm{s}$, which is very small. But if the channel switching happens when wireless devices are engaged in some communications, the upper layer protocols such as MAC-layer and routing-layer protocols will have a significant impact on the switching latency as we will show later.

Chandra et al. [14] study the concept of switching delay between multiple channels using only one 802.11 NIC through a software-based approach. They observe a delay of 3.9 seconds when switching from an infrastructure network to an ad hoc network and a delay of 2.8 seconds when switching from an ad hoc network to an infrastructure network. They reveal that the cause of this delay is the

1. The signal filter for transmitters is assumed to be the same as the transmission mask defined by IEEE 802.11 standard. More details can be found in [21]. 
media disconnect and media connect notifications to the IP stack. However, in our case, both the network ID (i.e., SSID) and the mode (i.e., ad hoc mode) are the same for the neighboring nodes that dynamically change the channel.

Vergetis et al. [30] also investigate the impacts of channel switching on the network throughput. Some degradations of the network performance can be observed, when the channel switches and only one NIC is used. In fact, their approach is to transmit packets across multiple channels over only a single hop (i.e., from a sender to an AP), while ours is to deliver packets using multiple channels over a multihop path, which is more practical in mesh networks.

Besides, the experiments in [14] and [30] are carried out with the use of laptops, while in our experiment, wireless routers, which have different software and hardware from laptops, are used because this is the case in WMNs.

\section{Experiment Configuration}

For demonstration purposes, we choose simple experiments to make our points. We are currently using IEEE-802.11compliant Linksys WRT54GL wireless routers operating in $b / g$ mode, which have been upgraded to run the Linuxbased OpenWRT OS. The available flash memory in our Linksys routers is 4 Mbytes, which is large enough to fit the OpenWRT OS. The Broadcom chipset is driven by a proprietary driver $(w l)$, which allows a reasonable degree of control over the wireless properties. Every router has a single wireless radio interface with two antennas for spatial diversity purposes and five wired interfaces. The default transmission power is $19 \mathrm{dBm}$. We have disabled the Request-To-Send/Clear-To-Send (RTS/CTS) mechanism and exploited the autorate adaptation provided by the Linksys routers. In order not to fall into the effects related to ground [10], we place the routers approximately at the height of $1 \mathrm{~m}$.

We collect the experimental data using Fujitsu notebooks of model P7010D, which are equipped with a 1.20-GHz Intel Pentium M processor and 512-Mbyte Random Access Memory (RAM). The notebooks run Linux 2.6 with a Debian distribution. In our experiments, we manually synchronize our nodes using ntpdate before each experiment to achieve millisecond timing accuracy. Note that ntpdate is an NTP [5] client that only synchronizes the local clock's phase offset to a common server (we use the gateway node as the NTP server), and it does not continuously track the timing error for the local clock's frequency offset. Because our experiments only last for a short period of time (several minutes at most), the local clock's frequency offset has little effect on the timing accuracy.

Besides, in our experiments, we use a free software called the Jugi's Traffic Generator (JTG) [2] to generate different kinds of traffics. More specifically, JTG can generate and inject different traffic patterns over TCP and/or UDP sockets. We decide to use JTG in our experiments since it can read the information on packet transmission intervals and packet sizes from files, allowing us to create an exact duplicate of a trace starting from a prerecorder stream. Traffic is then collected at the receiver side where suitable tools are available for analysis.

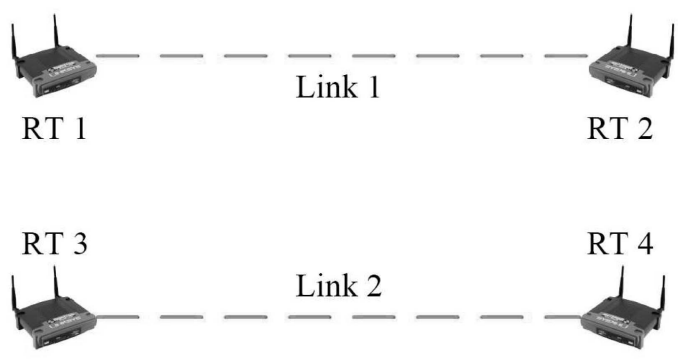

Fig. 2. Topology of the experiment on nonoverlapping channels.

\section{Experiments on ChanNel InTERFERENCE}

IEEE $802.11 \mathrm{a} / \mathrm{b} / \mathrm{g}$ standards specify many distinct frequency bands or channels, but most of them are overlapping. Specifically, the standards specify the central frequency of each channel, as well as a spectral mask that requires the signal to be attenuated by at least $30 \mathrm{~dB}$ from its peak energy at $11 \mathrm{MHz}$ from the center frequency and at least $50 \mathrm{~dB}$ at $22 \mathrm{MHz}$ from the center frequency, respectively. Since the central frequencies of two neighboring channels are $5 \mathrm{MHz}$ apart, two channels separated by at least five frequency bands can be considered to be nonoverlapping. Thus, IEEE $802.11 \mathrm{~b} / \mathrm{g}$ has up to three nonoverlapping channels, and IEEE 802.11a has up to 12 nonoverlapping channels.

Usually, it is commonly thought that the transmissions on two nonoverlapping channels will not interfere with each other. However, Liese et al. [22] and Robinson et al. [28] show by experiments that there does exist interference between nonoverlapping channels. In this section, we conduct some simple experiments to study the impacts of interchannel interference on network performance and to show whether it is possible for us to use multiple channels at the same time in WMNs.

The experiments are carried out on a playground in the University of Florida, and we make sure that our experiments do not have interferences from other APs. As shown in Fig. 2, there are four routers. Link 1 is set up between RT 1 and RT 2, and link 2 is set up between RT 3 and RT 4. The four routers all work in $b / g$ mode, and each router is connected to one laptop by an Ethernet cable, which acts as the traffic generator or receiver.

\subsection{Interference between Channel 1 and Channel 6}

We first check the impacts of the interference between channel 1 and channel 6 on network throughput. We set link 1 on channel 1 and link 2 on channel 6 . Two flows are set up on the two links, respectively. Flow 1 (the main flow) is from RT 1 to RT 2, and flow 2 (the interfering flow) is from RT 4 to RT 3. We use the Constant-Bit-Rate (CBR) traffic pattern for both flows and collect the throughput of flow 1 as its data sending rate increases from 1 to $11 \mathrm{Mbps}$ in four cases:

- Case 1: there is no traffic on link 2.

- Case 2: RT 4 is very close to RT 2, and the data sending rate of flow 2 is set to $1 \mathrm{Mbps}$.

- Case 3: RT 4 is $1 \mathrm{~m}$ away from RT 2, and the data sending rate of flow 2 is set to $1 \mathrm{Mbps}$.

- Case 4: RT 4 is very far from RT $2(15 \mathrm{~m})$, and the data sending rate of flow 2 is set to $11 \mathrm{Mbps}$. 


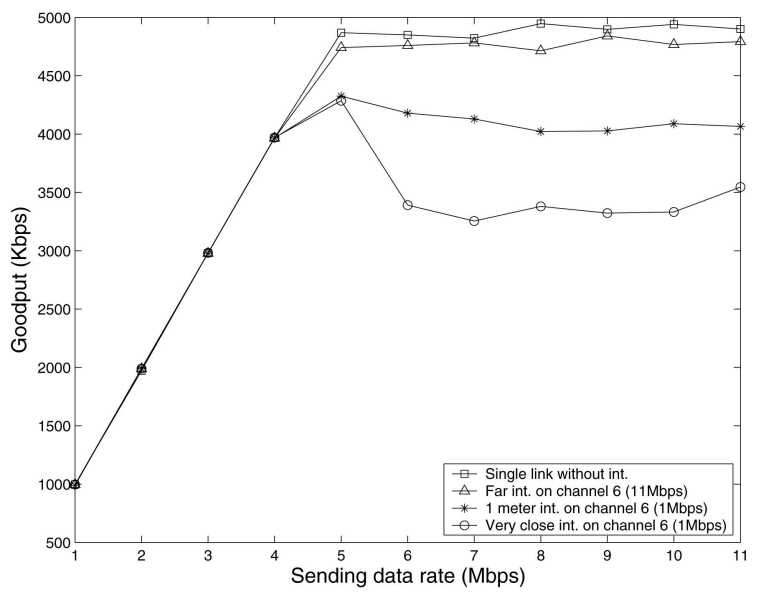

(a)

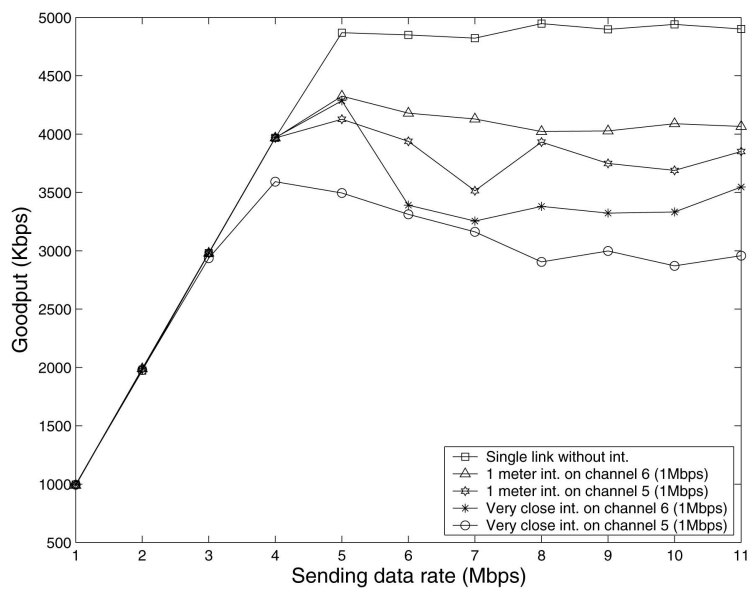

(b)

Fig. 3. UDP throughput at the receiver side of link 1. (a) Flow 2 is on channel 6. (b) Flow 2 is on channel 5.

The experimental results are shown in Fig. 3a. We observe that in Case 1, there is no interference, and the maximum throughput of flow 1 is about $5 \mathrm{Mbps}$; in Case 2 and Case 3, the maximum throughput of flow 1 degrades much when the data sending rate of flow 1 is more than $4 \mathrm{Mbps}$; in Case 4, the throughput of flow 1 is not affected much by flow 2 even when the data sending rate of flow 1 is as high as $11 \mathrm{Mbps}$.

\subsection{Interference between Channel 1 and Channel 5}

Notice that the center frequency of channel 1 is $25 \mathrm{MHz}$ away from that of channel 6 and $20 \mathrm{MHz}$ away from that of channel 5, which is a little smaller than that required in the standards. So obviously, the interference between channel 1 and channel 5 will be more severe than that between channel 1 and channel 6 . In this section, we check the impacts of the interference between channel 1 and channel 5 on network throughput.

We use the same topology and the same traffic pattern as that in Section 4.1, and we collect the throughput of flow 1 in case 2 and case 3 . The experiment results and comparisons with the previous case are shown in Fig. 3b. We can clearly observe that the throughput of flow 1 with interference on channel 5 is less than that of flow 1 with interference on channel 6 when the data sending rate of flow 1 is higher than 4 Mbps.

\subsection{Discussions}

From the results in Sections 4.1 and 4.2, we find that the transmissions on channel 5 or channel 6 do interfere with those on channel 1 . When the radios using different channels are far apart, the interference is not significant. When they are close to each other, the interference becomes noticeable. Besides, the interference between channel 1 and channel 5 or channel 6 can only be observed when the data sending rate of flow 1 is high enough.

Thus, we conclude that we can use multiple channels in WMNs only if either of the following holds:

1. The traffic in the neighborhood of a receiver is unsaturated, i.e., the following condition is satisfied:

$$
\sum_{i=1}^{n} r_{i}+r_{0} \leq \eta C,
$$

where $r_{i}$ is the data sending rate of the $i$ th $(1 \leq i \leq n)$ interfering neighbor of the receiver of interest (no matter which channel it is on), $r_{0}$ is the data sending rate of the particular flow under analysis, $C$ is the bandwidth of the channel the receiver is on, and $\eta(0<\eta<1)$ accounts for the communication overhead.

2. The radios using different channels are placed far apart from each other.

We notice that Mishra et al. have quantified in [24] the minimum distance required for two radios operating on two different channels not to interfere with each other. However, they assume that transmitters and receivers use the same signal filter, and they only use the free space power propagation model to predict the interference introduced by a transmitter at one receiver on another channel, which is not so practical. In the following, we derive the interference in a more practical and more general case.

Assume that node $i$ transmits to node $j$ on channel $x$, and node $j$ has some other neighboring transmitters denoted by the set $N(j)$. For node $k \in N(j)$, it operates on channel $c_{k}$. Recall the general model in [27] used to predict the received power at a receiver that is on the same channel as a transmitter, i.e.,

$$
P_{r}(d)=P_{t} h\left(G_{t}, G_{r}, h_{t}, h_{r}, L, \lambda\right) \frac{1}{d^{\alpha}},
$$

where $P_{t}$ and $P_{r}$ are the transmitted power and the received power, respectively, $G_{t}$ and $G_{r}$ are the gain factors for the transmitter antenna and the receiver antenna, respectively, $h_{t}$ and $h_{r}$ are the antenna heights of the transmitter and the receiver, respectively, $d$ is the distance between the transmitter and the receiver, $L$ is the system loss factor not related to propagation $(L \geq 1), \lambda$ is the wavelength, $h(\cdot)$ is a function, and $\alpha$ is the path loss exponent. Then, denoting by $d(j, k)$ the distance between node $k$ and node $j$ and by $P_{t, k}$ and $P_{r, j}\left(d(j, k), x, c_{k}\right)$ the transmitted power of node $k$ and the received power at node $j$, respectively, as we have shown in [21], the interference introduced by node $k$ to node $j$ can be represented by 


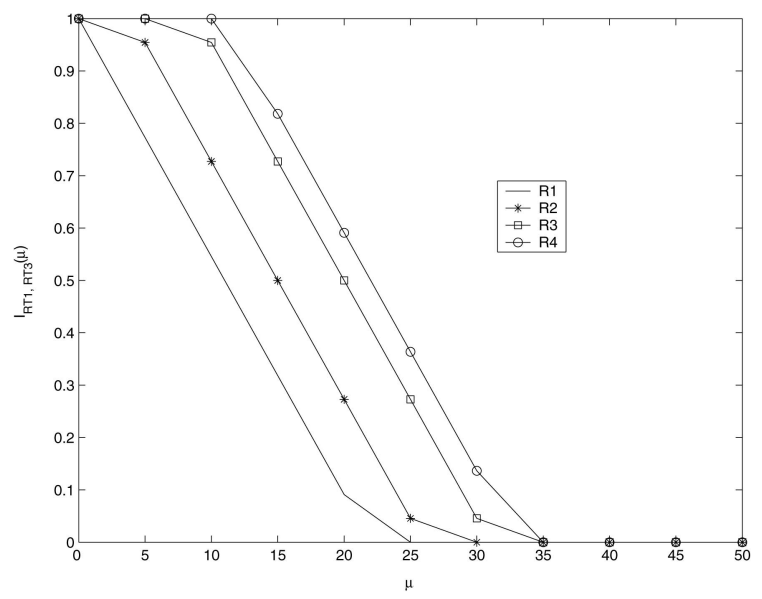

Fig. 4. The values of $I(\mu)$ for four different receiver filters denoted by R1, $\mathrm{R} 2$, R3, and R4, respectively.

$$
P_{r, j}\left(d(j, k), x, c_{k}\right)=P_{t, k} h\left(G_{t}, G_{r}, h_{t}, h_{r}, L, \lambda\right) \frac{I\left(x, c_{k}\right)}{d(j, k)^{\alpha}},
$$

where $I\left(x, c_{k}\right)\left(0 \leq I\left(x, c_{k}\right) \leq 1\right)$ captures the interference between channel $x$ and channel $c_{k}$.

As we have shown in [21], the values of $I(\mu)=I\left(x, c_{k}\right)=$ $\int_{-\infty}^{\infty} S_{k}(f) Z_{r, j}(f-\mu) d f$, where $S_{k}(f)$ is the transmitted signal of node $k, Z_{r, j}(f)$ denotes the band-pass filter's frequency response of node $j$, and $\mu=5\left|x-c_{k}\right|$, can be represented in Fig. 4, for four different receiver filters denoted by R1, R2, $\mathrm{R} 3$, and R4, respectively, i.e.,

- (R1): the receiver filter is the same as the transmit spectrum mask. $^{2}$

- (R2): the receiver filter is a band-pass filter with a bandwidth of $30 \mathrm{MHz}$.

- (R3): the receiver filter is a band-pass filter with a bandwidth of $40 \mathrm{MHz}$.

- (R4): the receiver filter is a band-pass filter with a bandwidth of $44 \mathrm{MHz}$.

In order for node $j$ to correctly receive the signal from node $i$, two conditions should be satisfied: 1 ) the received desired signal is greater than the receiver sensitivity (denoted by $\left.R X_{t h}\right)$, and 2) the signal-to-noise-plus-interference ratio (SNIR) is above a threshold (denoted by $S I N R_{t h}$ ) in the presence of interference. Usually, condition 1 is satisfied. Then, the only requirement is condition 2 , i.e.,

$$
\frac{P_{r, j}(d(j, i))}{P_{\text {thermal }}+\sum_{k \in N(j)} P_{r, j}\left(d(j, k), x, c_{k}\right)} \geq S I N R_{t h},
$$

where $P_{\text {thermal }}$ is the strength of the thermal noise, $P_{r, j}(d(j, i))$ is the power from the transmitter $i$ at distance $d(j, i)$, and $P_{r, j}\left(d(j, k), x, c_{k}\right)$ is given by (2).

Substituting (2) into (3), we can obtain requirements on distances $d(j, k)(k \in N(j))$ for node $j$ to successfully receive packets from node $i$. Furthermore,

$$
\frac{P_{r, j}(d(j, i))}{P_{\text {thermal }}+P_{r, j}\left(d(j, k), x, c_{k}\right)} \geq S I N R_{t h}
$$

2. The transmit spectrum mask can be found in [21].

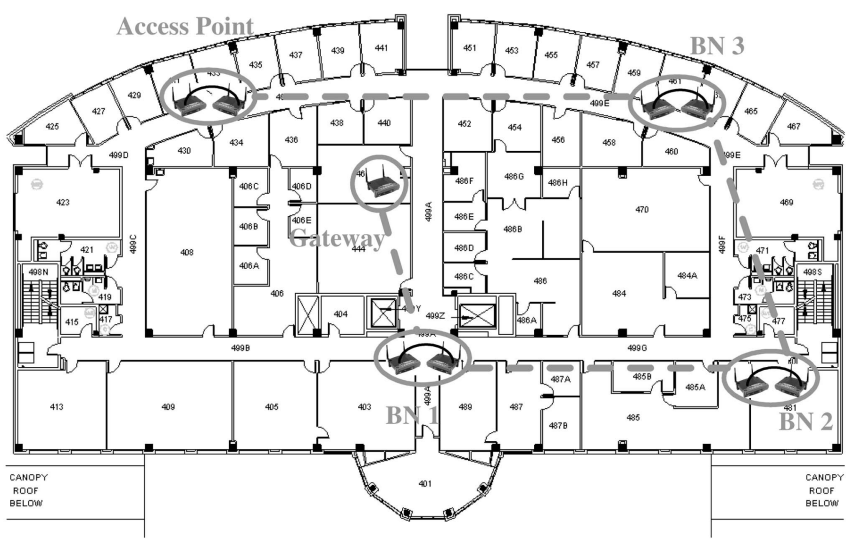

Fig. 5. WMN testbed placement (fourth floor in the New Engineering Building, University of Florida).

gives a lower bound on the minimum distance required for node $k$ not to interfere with node $j$ 's reception, i.e., when node $k$ is the only interfering transmitter of node $j$.

\section{Experiments on Channel Switching Cost}

In Section 4, we have shown by experiments that it is still feasible to use multiple channels in WMNs under certain conditions. In this section, we turn to the question Q2: what is the cost we have to pay when we switch channels at the mesh routers in a WMN? Here, "cost" refers to the end-to-end delay, the packet loss ratio, and the channel switching time. Moreover, we also investigate the relationship between the cost and the number of hops on a path where the channels are changing. Since the number of hops from the end users to the gateway is usually very small, for example, it is fewer than five hops on the average in MIT Roofnet [12], we develop experiments with backbone links (BLs) ranging from one to four hops.

For this particular experiment, we adopt a three-tier chain topology [13] to set up our testbed. It consists of one gateway, a backbone network formed by three BNs, and one AP. As shown in Fig. 5, this testbed is located on the fourth floor of the New Engineering Building at the University of Florida, simulating a typical office environment. Each wireless BN is composed of two routers attached to each other via an Ethernet cable. These two routers are configured on two different channels to form a $\mathrm{BN}$ with two radios and two channels. Besides, the routers in the BNs are set to run in ad hoc mode, while the AP operates in master mode (also called infrastructure mode). We attach the AP via an Ethernet cable to another wireless router operating in ad hoc mode so that the AP can be connected to the backbone network and the gateway. Moreover, each router runs the OLSR protocol such that the network can be easily extended.

The topology of our testbed is shown in Fig. 6. Although it is a small testbed, it is sufficient for us to make our points. We connect a transmitter to the gateway via an Ethernet cable and a receiver to the AP via wireless medium, respectively. Then, we generate Voice-over-IP (VoIP) and video traffics and transmit them through this chain. The reason why we choose VoIP and video traffics is twofold. First, they are common traffics in mesh networks. Second, they are typical traffics: VoIP traffics have low data rates (in kilobits per second) and low ratio between the peak bit rate and average bit rate, while 


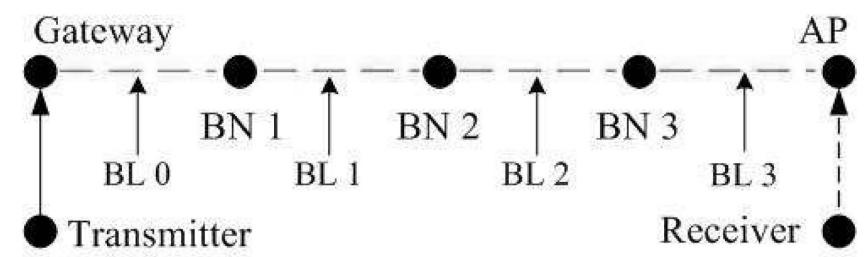

Fig. 6. The topology of our test-bed.

video traffics have high data rates (in megabits per second) and high peak-to-average bit rate ratio. Each BL in Fig. 6 uses a different channel chosen from $[1,10]$ and changes the channel every 1 minute. On the other hand, the AP uses channel 11 and never changes its channel. We develop a simple channel assignment algorithm to assign channels for the BLs:

$$
\text { channel }_{t+1}^{i}=\left(\text { channel }_{t}^{i}+4\right) \bmod 10+1,
$$

where channel $_{t}^{i}$ and channel ${ }_{t+1}^{i}$ are the channels of BL $i$ at the $t$ th minute and the $(t+1)$ th minute, respectively, and $0 \leq i \leq 3$. Besides, we initially set channel $_{1}^{0}=1$, channel $_{1}^{1}=5$, channel $_{1}^{2}=9$, and channel ${ }_{1}^{3}=3$, and the complete channel assignment is shown in Table 1. There are two reasons for choosing this algorithm:

- First, we find that it is impossible to select a step less than four when we try to switch channels for these Linksys routers.

- Second, this algorithm is very simple, but it is sufficient for us to investigate the cost of channel switching.

The traffics last for 10 minutes, which means that each BL will change the channel nine times during this period. After the transmission finishes, we will have a log file in the receiver, recording a tuple of information for every received packet: the sequence number, the transmitting time, the receiving time, and the packet size. Based on this information, we can calculate the end-to-end delay of each received packet and the packet loss ratio. We repeat this procedure four times, changing the BLs from one to four hops. In each case, we compare the performance of the network when the BLs keep switching with that when the BLs are static, i.e., they never change their channels.

\subsection{The Case of Audio Traffics}

Many important voice applications employ CBR coding, e.g., Skype and softphones. However, several voice codecs can optionally employ Voice Activity Detection (VAD), a technique typically used in speech processing that aims at detecting the presence or absence of human speech. Under

TABLE 1

Channel Assignments

\begin{tabular}{|c|c|c|c|c|}
\hline Time & BL0 & BL1 & BL2 & BL3 \\
\hline Minute 1 & Channel 1 & Channel 5 & Channel 9 & Channel 3 \\
\hline Minute 2 & Channel 6 & Channel 10 & Channel 4 & Channel 8 \\
\hline Minute 3 & Channel 1 & Channel 5 & Channel 9 & Channel 3 \\
\hline Minute 4 & Channel 6 & Channel 10 & Channel 4 & Channel 8 \\
\hline Minute ... & Channel ... & Channel ... & Channel ... & Channel ... \\
\hline
\end{tabular}

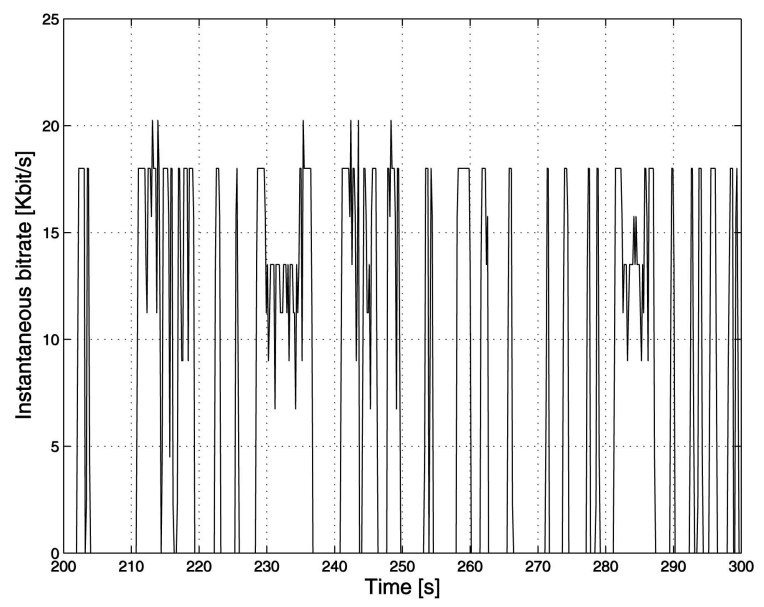

Fig. 7. Instantaneous bit rate of a GSM 6.10 encoded voice trace with VAD enabled.

VAD, the application stops transmitting packets when the user is not talking until new voice activity is detected. Clearly, since the VAD packet source generates packets only during active periods, consistent bandwidth savings are possible.

We conduct our experiments under VAD-enabled voice traffic. Traffic traces have been generated using Ekiga [1], an open source VoIP and videoconferencing application. At one end, we register voice traces corresponding to a VAD-enabled GSM 6.10 device, dumping the resulting packet trace with WireShark [7]. The instantaneous bit rate of the recorded voice trace pattern over a 100-second interval is reported in Fig. 7, where we can clearly distinguish the active and silent periods modulated by $\mathrm{VAD}$. In the VAD-enabled case, according to our voice recorded traces, the codec is detected inactive for a fraction of time $a=53$ percent.

The experimental results of the end-to-end delay of the audio traffic are shown in Fig. 8. We can clearly see that there are some impulses when the channels are changing. This indicates that when the BL switches from one channel to another, the end-to-end delay of packets increases drastically. Since this figure only shows the end-to-end delay of the received packets, we dig out how many packets are lost due to the channel switching in the following.

Let $F_{L}(t)$ and $f_{L}(t)$ denote the cumulative distribution function and the probability density function of packet loss, respectively. First, we further define $F_{L}^{i}(t)$ as

$F_{L}^{i}(t)=$ the cumulative distribution function of lost packets during $[\mathrm{i}, \mathrm{i}+1]$ minutes, for $t \in[1,60]$ seconds,

where $i=1,2,3, \ldots, 9$.

Then, we calculate $F_{L}(t)$ and $f_{L}(t)$ by

$$
\begin{aligned}
F_{L}(t) & =\sum_{i=1}^{9} F_{L}^{i}(t) / 9, \\
f_{L}(t) & =\frac{d}{d t} F_{L}(t) .
\end{aligned}
$$

The experimental results of the packet loss of audio traffics are shown in Fig. 9. 


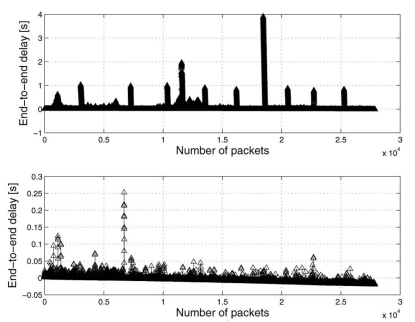

(a)

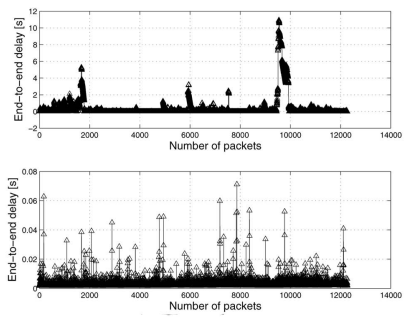

(b)

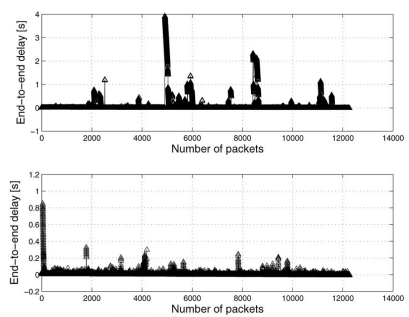

(c)

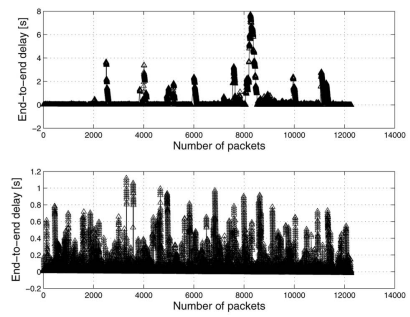

(d)

Fig. 8. End-to-end delay of audio traffic (Upper: dynamic channel switching; lower: static channel, no channel switching). (a) One hop. (b) Two hops. (c) Three hops. (d) Four hops.

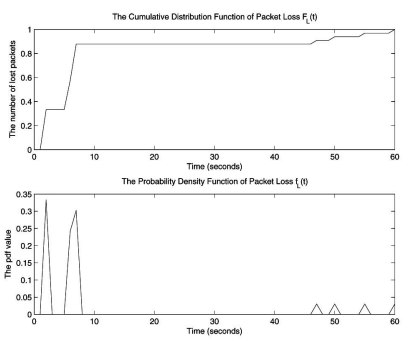

(a)

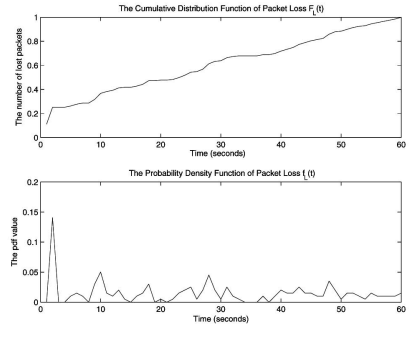

(b)
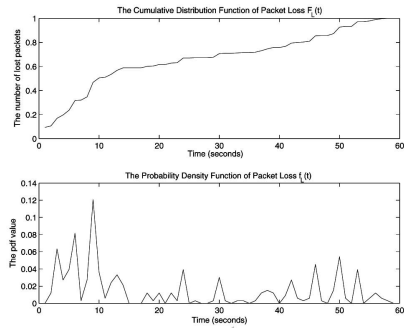

(c)
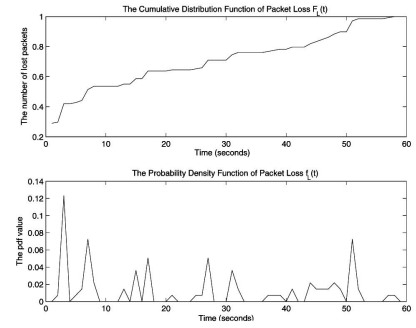

(d)

Fig. 9. Packet loss of audio traffic (Upper: cumulative distribution function of packet loss; lower: probability density function of packet loss). (a) One hop. (b) Two hops. (c) Three hops. (d) Four hops.

In addition to the end-to-end delay and the packet loss ratio, we also calculate the Mean Opinion Score (MOS), which is traditionally used to assess the quality of conversation in VoIP systems. MOS is a numerical measure and is expressed as a single number in the range 1 to 5 , where 1 is the lowest perceived quality, and 5 is the highest perceived quality. Since it is based on a listening test, evaluating the MOS rate for a VoIP solution can be a time-consuming process. For this reason, we make our probes through synthetic traffic generation, and we resort to the E-Model [3], which provides an objective method to evaluate speech quality in VoIP systems (please refer to [16] and [18] for a thorough description). The outcome of an E-Model evaluation is called the R-factor $(R)$, which is a numerical measure of voice quality, ranging from 0 to 100 . The reference values of the $\mathrm{R}$-factor are categorized as shown in Table 2.

In the E-Model, several different parameters affecting the quality of a conversation are taken into account. The main assumption is that various impairments at the physiological scale have an additive behavior (decibel-like behavior):

$$
R=R_{0}-I_{s}-I_{d}-I_{e}+A .
$$

TABLE 2

R-Factors, Quality Ratings, and the Associated MOS

\begin{tabular}{|c|c|c|}
\hline R-factor & Quality of voice rating & MOS \\
\hline \hline $90<R \leq 100$ & Best & $4.34-4.5$ \\
\hline $80<R \leq 90$ & High & $4.03-4.34$ \\
\hline $70<R \leq 80$ & Medium & $3.60-4.03$ \\
\hline $60<R \leq 70$ & Low & $3.10-3.60$ \\
\hline $50<R \leq 60$ & Poor & $2.58-3.10$ \\
\hline
\end{tabular}

In particular, $R_{0}$ is the basic signal-to-noise ratio (environmental and device noises), $I_{s}$ accounts for the impairments on the coded voice signal (loud connection and quantizations), $I_{d}$ represents the effect of delay, $I_{e}$ represents the effect of low-bit-rate codecs, and $A$ is the advantage factor, corresponding to the user allowance due to the convenience in using a given technology. We reported in Table 3 some sample values for the R-factor for different scenarios.

The main advantage of the E-model is that for a given codec, i.e., given $I_{e}$, only delays and losses are needed for speech quality estimation.

According to [16], (5) can be further simplified into the following expression:

$$
R=93.4-I_{d}\left(T_{a}\right)-I_{e f}(\text { codec }, \text { loss_rate }) .
$$

The relation between $I_{d}$ and the one-way delay $T_{a}$ is expressed as

$$
I_{d}=0.024 T_{a}+0.11\left(T_{a}-177.3\right) H\left(T_{a}-177.3\right),
$$

TABLE 3

The Typical R-Factor Values of Some Reference Cases

\begin{tabular}{|c|c|}
\hline Scenario & R \\
\hline \hline PSTN/PSTN & 82 \\
\hline ISDN/ISDN & 92 \\
\hline PSTN/Mobile & 64 \\
\hline VoIP & 68 \\
\hline
\end{tabular}




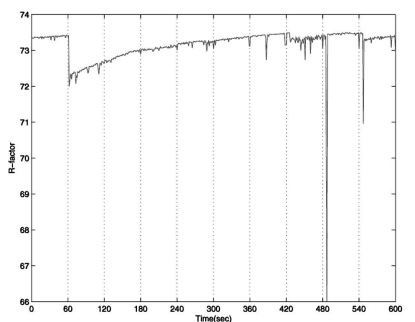

(a)

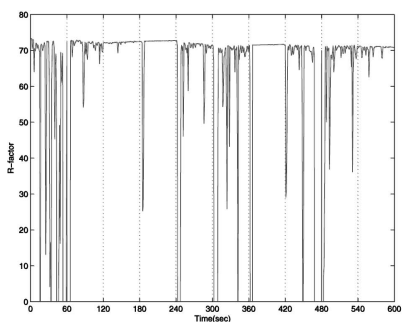

(b)

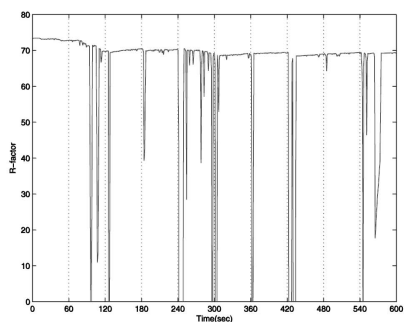

(c)

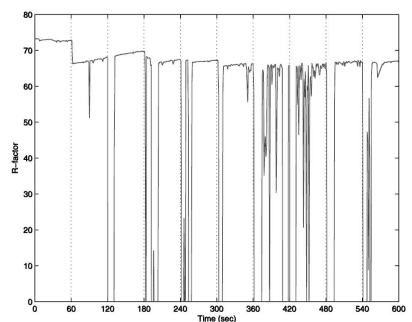

(d)

Fig. 10. Instantaneous R-factor of audio traffic. (a) One hop. (b) Two hops. (c) Three hops. (d) Four hops.

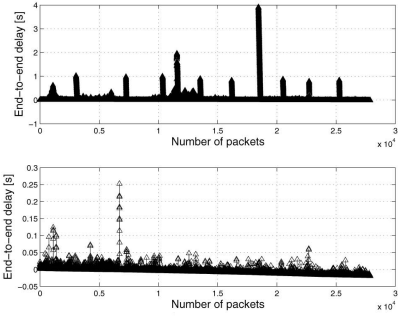

(a)

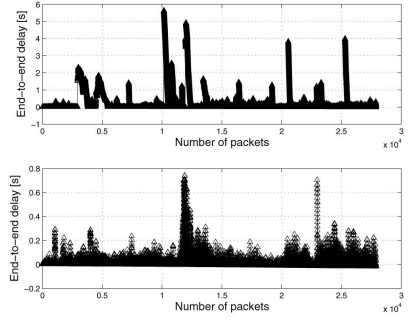

(b)

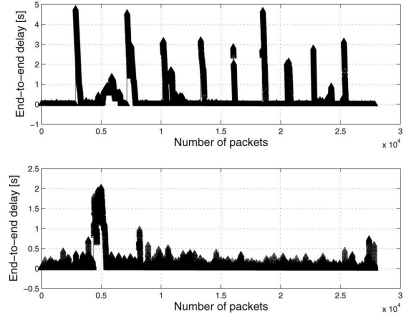

(c)

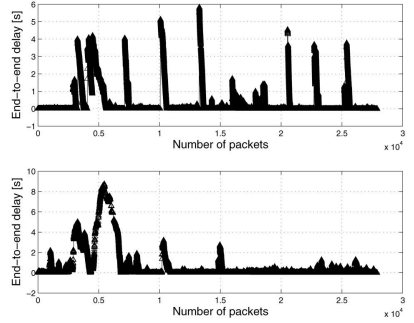

(d)

Fig. 11. End-to-end delay of video traffic (Upper: dynamic channel switching; lower: static channel, no channel switching). (a) One hop. (b) Two hops.

(c) Three hops. (d) Four hops.
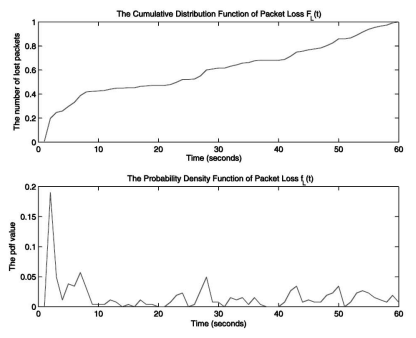

(a)
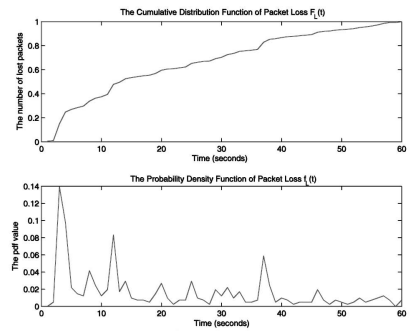

(b)
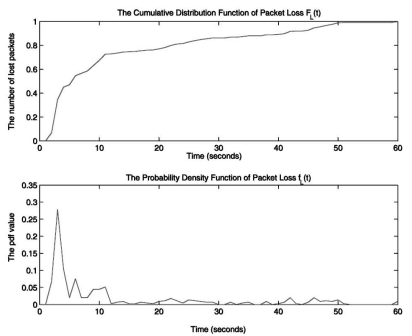

(c)
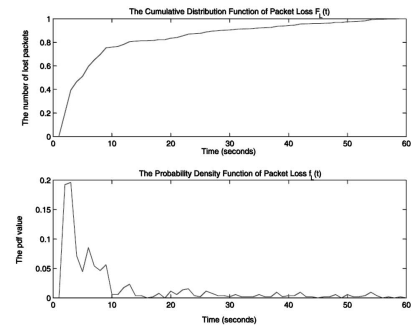

(d)

Fig. 12. Packet loss of video traffic (Upper: cumulative distribution function of packet loss; lower: probability density function of packet loss). (a) One hop. (b) Two hops. (c) Three hops. (d) Four hops.

where $H(x)$ is the step function. $I_{e f}$ in (6) is the equipment impairment (nonlinear codecs and packet losses), which can be calculated as $[4]^{3}$

$$
I_{e f}=I_{e_{\text {opt }}}+C_{1} \ln \left(1+C_{2} \cdot \text { loss_rate }\right) \text {. }
$$

In our tests, we adopt the GSM 6.10 codec, so the formula for the impairment factor is given as

$$
I_{e f}=I_{e_{o p t}}+\left(95-I_{e_{\text {opt }}}\right) \frac{\text { loss_rate }}{\text { loss_rate }+B_{p l}},
$$

where $B_{p l}$ is the packet loss robustness factor of the audio codec [20]. Notice that $I_{e_{\text {opt }}}, C_{1}, C_{2}$, and $B_{p l}$ shown above are all codec-specific parameters. For the calculation of (7), we use $I_{e_{\text {opt }}}=20$ and $B_{p l}=43$.

The instantaneous values of the R-factor are shown in Fig. 10. We observe that the R-factor value decreases significantly when the channel is switching.

3. The calculation is accurate up to loss_rate $=0.1$; for higher values, it may prove optimistic.

\subsection{The Case of Video Traffics}

We also perform some experiments on the support of video traffics offered by the WMN testbed under the condition of dynamically changing the channel. These experiments aim at probing the system in case of a VBR traffic with a large ratio of the peak bit rate to the average bit rate, accounting also for background FTP traffic. In particular, we considered a sample video trace, namely, the Silence of the Lambs, an MPEG4 video encoded with rate control and single-layer encoding publicly available in [6]. The stream is encoded with a target rate of $64 \mathrm{Kbps}$, an average bit rate of $226,852 \mathrm{bps}$, and a peak rate of $2.951040 \mathrm{Mbps}$. Since the plain frame sequence consists of frames of up to 2,800 bytes, we preprocessed the traces in order to packetize the flow with an MPDU of 1,000 bytes. ${ }^{4}$

The experimental results of the end-to-end delay of the video traffics are shown in Fig. 11. We can clearly observe that there are some impulses when the channels are changing.

4. More sophisticated packetization of video frames would require, in principle, to account for the underlying coding, so that single-packet losses do not prevent partial frame utilization. 
TABLE 4

Packet Loss Ratio for VolP Traffics with Channel Switching

\begin{tabular}{|c|c|c|c|c|}
\hline Packet loss ratio & One hop & Two hops & Three hops & Four hops \\
\hline$P_{c s}$ & $0.34 \%$ & $0.59 \%$ & $0.96 \%$ & $2.86 \%$ \\
\hline$P_{T}$ & $1.61 \%$ & $1.82 \%$ & $2.75 \%$ & $4.46 \%$ \\
\hline$P_{c s} / P_{T}$ & $21.1 \%$ & $32.4 \%$ & $34.9 \%$ & $64.1 \%$ \\
\hline
\end{tabular}

Following the process in Section 5.1, we can obtain the packet loss of the video traffic, which is shown in Fig. 12.

In Fig. 9 and Fig. 12, we can see that packets are mainly lost in the first 10 seconds. We hence conclude that it takes up to 10 seconds for the BL to reach the steady state again after changing from one channel to another. In other words, the channel switching time is up to 10 seconds when the number of hops on which the channel is changing is up to five.

Next, we define the total packet loss ratio $P_{T}$ and the packet loss ratio due to channel switching $P_{c s}$ as follows:

$$
\begin{aligned}
P_{T} & =\frac{\# \text { of lost packets }}{\text { the total number of transmitted packets }}, \\
P_{c s} & =\frac{\# \text { lost packets due to channel switching }}{\text { the total number of transmitted packets }} .
\end{aligned}
$$

Let $N_{c s}^{i}$ denote the number of lost packets in the first 10 seconds in $[\mathrm{i}, \mathrm{i}+1]$ minutes and $N$ denote the total number of transmitted packets in 10 minutes. We can obtain

$$
P_{c s} \approx \frac{\sum_{i=1}^{9} N_{c s}^{i}}{N}
$$

The numerical results of the packet loss ratio for VoIP traffics and video traffics when the channels are switching are shown in Table 4 and Table 5, respectively. We find that $P_{c s}$ increases as the number of hops increases, and so does $P_{c s} / P_{T}$. Noticeably, the ratio of $P_{c s}$ to $P_{T}$ even increases to more than 60 percent when the number of backbone hops is four. This indicates that the cost of channel switching in terms of packet loss ratio increases as the number of hops increases. We also show in Table 6 the total packet loss ratios when the channels are static. Comparing the results in Table 4 and Table 5 with those in Table 6, we observe that when the BLs keep switching the channel every 1 minute, more packets are lost compared to those in the case where the BLs use static channels.

As a result, we recommend that we should not change channels too often and should carefully determine when to change the channel. Moreover, the channel assignment schemes selecting one channel for each packet may not be worthwhile because the cost due to channel switching may be too high.

TABLE 5

Packet Loss Ratio for Video Traffics with Channel Switching

\begin{tabular}{|c|c|c|c|c|}
\hline Packet loss ratio & One hop & Two hops & Three hops & Four hops \\
\hline$P_{c s}$ & $0.4 \%$ & $0.54 \%$ & $1.08 \%$ & $1.4 \%$ \\
\hline$P_{T}$ & $1.18 \%$ & $1.44 \%$ & $1.81 \%$ & $2.08 \%$ \\
\hline$P_{c s} / P_{T}$ & $33.9 \%$ & $37.5 \%$ & $59.6 \%$ & $67.3 \%$ \\
\hline
\end{tabular}

TABLE 6

Packet Loss Ratio for Video Traffic with Static Channel

\begin{tabular}{|c|c|c|c|c|}
\hline Packet loss ratio & One hop & Two hops & Three hops & Four hops \\
\hline$P_{\text {voip }}$ & $0.09 \%$ & $0.24 \%$ & $0.43 \%$ & $1.35 \%$ \\
\hline$P_{\text {video }}$ & $0.2 \%$ & $0.39 \%$ & $1.35 \%$ & $1.45 \%$ \\
\hline
\end{tabular}

\section{Several Factors Related to Channel SWITCHING COST}

So far, we have investigated the channel switching cost in WMNs using only Linksys WRT54GL wireless routers with the OLSR protocol. In this section, we study the impacts of routing protocols and hardware on the channel switching cost.

\subsection{Impacts of Routing Protocols}

In our experiments, we assume that every link switches the channel once a minute. This is actually performed by making the two routers on this link to switch to the same channel at the same time. However, as we all know, it is very difficult to achieve accurate synchronization in wireless networks and the time for two routers to switch the channel is not necessarily exactly the same. Thus, every time a link switches the channel, there is a period during which the link is "down" and the route discovery process at the routing layer will be activated. As a result, there is no surprise that many packets are lost at the beginning of channel switching.

In order to show the impacts of routing protocols, we carry out again the experiments shown before using static routing instead of the OLSR protocol. By using static routing, no route discovery process is needed because we can manually set up the routing table in each router to maintain a path from the transmitter to the receiver.

The packet losses of video traffic transmitted through the chain topology with three hops and four hops are shown in Fig. 13a and Fig. 13b, respectively, and the numerical results of the packet loss ratio are shown in Table 7. Compared with the results with the OLSR protocol, i.e., Fig. 12c and Fig. 12d and Table 5, we observe that the channel switching time is reduced from about 10 seconds (with the OLSR protocol) to about 6 seconds (with the static routing), and the packet loss ratio due to channel switching is also decreased. However, the channel switch cost is still significant in terms of its magnitude.

\subsection{Impacts of Hardware}

Note that all the experiments above are performed using Linksys WRT54GL wireless routers. Here, we turn to study the impacts of hardware on channel switching cost by choosing another kind of widely used wireless router, i.e., Netgear WGT634U wireless router, to carry out some experiments. We use the same topology, the same routing protocol, and the same traffic patterns as those in Section 6.1.

The packet losses of video traffic transmitted through the chain topology with three hops and four hops are shown in Fig. 13c and Fig. 13d, respectively. We can clearly see that the channel switching time is about 3 seconds in the threehop case and about 6 seconds in four-hop case, respectively. Compared with the results obtained from using Linksys wireless routers, i.e., Fig. 13a and Fig. 13b, we can find that the channel switching time using Netgear wireless routers is a little bit shorter than that using Linksys routers, but it is still as high as several seconds, which is still significant. 

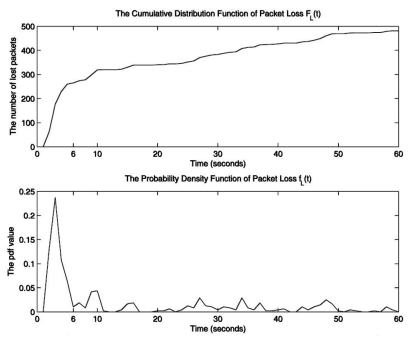

(a)

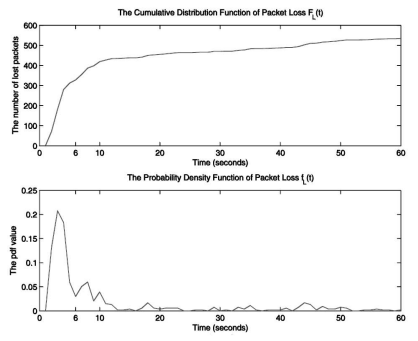

(b)
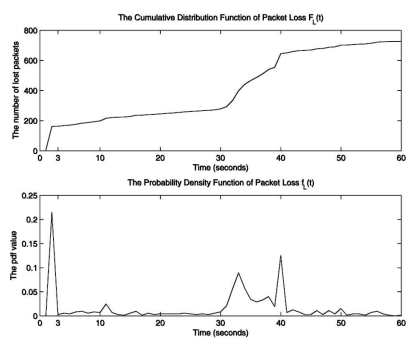

(c)
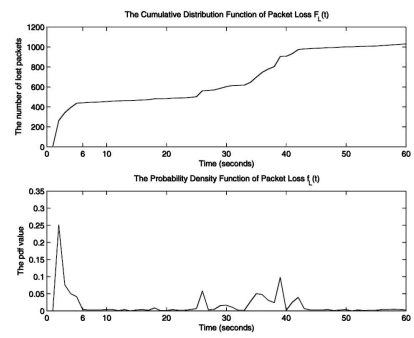

(d)

Fig. 13. Packet loss of video traffic with static routing. Upper: cumulative distribution function of packet loss; lower: probability density function of packet loss. (a) Three hops (Linksys routers). (b) Four hops (Linksys routers). (c) Three hops (Netgear routers). (d) Four hops (Netgear routers).

Thus, we conclude that the change of routing protocols or hardware cannot significantly reduce the channel switching cost. In other words, the conclusion in Section 5 still holds, and we should avoid switching channels frequently.

\section{ConCLUSIONS}

In this paper, we study the feasibility and the impacts of using multiple channels in WMNs by conducting some real experiments. We have shown that there is indeed some interference between nonoverlapping channels, as well as partially overlapping channels. However, we can still efficiently use multiple channels in the network if either the traffic in the neighborhood of each receiver is unsaturated or the radios using different channels are placed far apart from each other. Thus, those proposed multichannel MAC protocols should be reconsidered with these additional considerations.

We also carry out some experiments to show the cost of dynamically switching channels. We observe that when the number of hops on a path increases, the channel switching time can reach a few seconds, during which both the endto-end delay and the packet loss ratio increase significantly. The use of static routing can reduce the channel switching time due to smaller control overheads, but the channel switching time is still in seconds and still too costly. Although using another hardware may reduce the channel switching cost, unfortunately, the difference is not significant. As a result, when designing the channel assignment algorithms, we should not switch the channels too frequently. Especially, those per-packet channel (re)assignment algorithms are not really recommended due to the high channel switching cost. Besides, the design of protocols at various layers to reduce channel switching cost will be a very interesting and challenging problem, which will be investigated in the future.

Furthermore, in our experiments, we use the most basic scheme to cache and retransmit packets. We will also investigate the impacts of caching and retransmitting schemes on the network performance in our future research.

TABLE 7

Packet Loss Ratio for Video Traffic with Channel Switching (Static Routing)

\begin{tabular}{|c|c|c|}
\hline Packet loss ratio & Three hops & Four hops \\
\hline$P_{c s}$ & $0.95 \%$ & $1.17 \%$ \\
\hline$P_{T}$ & $1.73 \%$ & $1.91 \%$ \\
\hline$P_{c s} / P_{T}$ & $54.9 \%$ & $61.3 \%$ \\
\hline
\end{tabular}

\section{ACKNOWLEDGMENTS}

This work was partially supported by the USNational Science Foundation under Grants CNS-0721744 and DBI-0529012. The work of Yuguang Fang was also partially supported by the 111 Project under B08038 with Xidian University, China, and the work of Enrico Gregori was also partially supported by the Italian National Project: Wireless multiplatfOrm mimo active access netwoRks for QoS-demanding muLtimedia Delivery (WORLD), under grant number 2007R989S.

\section{REFERENCES}

[1] Ekiga, http://www.gnomemeeting.org, 2008.

[2] Jtg. https://hoslab.cs.helsinki.fi/savane/projects/jtg/, 2008.

[3] Recommendation G.107 : The E-Model, A Computational Model for Use in Transmission Planning, 2008.

[4] Recommendation G.113: Transmission Impairments Due to Speech Processing, 2000.

[5] Simple Network Time Protocol (SNTP) Version 4, http://www.apps. ietf.org/rfc/rfc2030.html, 2008.

[6] Video Traces Research Group, http://trace.eas.asu.edu/, 2008.

[7] Wireshark, http://www.wireshark.org, 2008.

[8] I. Akyildiz, W. Su, Y. Sankarasubramaniam, and E. Cayirci, "Wireless Sensor Networks: A Survey," Elsevier Computer Networks, vol. 38, no. 4, pp. 392-422, Mar. 2002.

[9] M. Alicherry, R. Bhatia, and L.L. Li, "Joint Channel Assignment and Routing for Throughput Optimization in Multiradio Wireless Mesh Networks," IEEE J. Selected Areas in Comm., vol. 24, no. 11, pp. 1960-1971, Nov. 2006.

[10] A. Anastasi, E. Borgia, M. Conti, E. Gregori, and A. Passarella, "Understanding the Real Behavior of Mote and 802.11 Ad Hoc Networks: An Experimental Approach," Pervasive and Mobile Computing, Mar. 2005.

[11] P. Bahl, R. Chandra, and J. Dunagan, "SSCH: Slotted Seeded Channel Hopping for Capacity Improvement in IEEE 802.11 Ad-Hoc Wireless Networks," Proc. ACM MobiCom '04, Sept. 2004.

[12] J. Bicket, D. Aguayo, S. Biswas, and R. Morris, "Architecture and Evaluation of an Unplanned 802.11b Mesh Network," Proc. ACM MobiCom'05, Aug. 2005.

[13] R. Bruno, M. Conti, and E. Gregori, "Mesh Networks: Commodity Multihop Ad Hoc Networks," IEEE Comm. Magazine, vol. 43, no. 3, pp. 123-131, Mar. 2005.

[14] R. Chandra, P. Bahl, and P. Bahl, "MultiNet: Connecting to Multiple IEEE 802.11 Networks Using a Single Wireless Card," Proc. IEEE INFOCOM '04, Mar. 2004.

[15] N. Choi, Y. Seok, and Y. Choi, "Multi-Channel MAC Protocol for Mobile Ad Hoc Networks," Proc. 58th IEEE Vehicular Technology Conf. (VTC-Fall '03), Oct. 2003.

[16] R.G. Cole and J.H. Rosenbluth, "Voice over IP Performance Monitoring," Proc. ACM SIGCOMM '01, Aug. 2001.

[17] F. Herzel, G. Fischer, and H. Gustat, "An Integrated CMOS RF Synthesizer for 802.11a Wireless LAN," IEEE J. Solid-State Circuits, Oct. 2003.

[18] C. Hoene, H. Karl, and A. Wolisz, "A Perceptual Quality Model Intended for Adaptive VoIP Applications: Research Articles," Int'l J. Comm. Systems, vol. 19, no. 3, pp. 299-316, 2006. 
[19] R. Huang, H. Zhai, C. Zhang, and Y. Fang, "SAM-MAC: An Efficient Channel Assignment Scheme for Multi-Channel Ad Hoc Networks," Computer Networks: The Int'l J. Computer and Telecomm. Networking, vol. 52, no. 8, pp. 1634-1646, 2008.

[20] R. Kwitt, T. Fichtel, and T. Pfeiffenberger, "Measuring Perceptual VoIP Speech Quality over UMTS," Proc. Fourth Int'l Workshop Internet Performance, Simulation, Monitoring, and Measurement (IPS-MoMe), 2006.

[21] P. Li, N. Scalabrino, Y. Fang, E. Gregori, and I. Chlamtac, "Channel Interference in IEEE 802.11b Systems," Proc. IEEE GLOBECOM '07, Nov. 2007.

[22] S. Liese, D. Wu, and P. Mohapatra, "Experimental Characterization of an 802.11b Wireless Mesh Network," Technical Report TR-CSE-2005-17, 2008

[23] A. Mishra, E. Rozner, S. Banerjee, and W. Arbaugh, "Exploiting Partially Overlapping Channels in Wireless Networks: Turning a Peril into an Advantage," Proc. ACM/Usenix Internet Measurement Conf. (IMC'05), Oct. 2005.

[24] A. Mishra, V. Shrivastava, S. Banerjee, and W. Arbaugh, "Partially Overlapped Channels Not Considered Harmful," Proc. ACM SIGMETRICS '06, June 2006.

[25] A. Raniwala and T. Chiueh, "Architecture and Algorithms for an IEEE 802.11-Based Multi-Channel Wireless Mesh Network," Proc. IEEE INFOCOM '05, Mar. 2005.

[26] A. Raniwala, K. Gopalan, and T. Chiueh, "Centralized Algorithms for Multi-Channel Wireless Mesh Networks," ACM Mobile Computing and Comm. Rev., vol. 8, no. 2, pp. 50-65, Apr. 2004.

[27] T. Rappaport, Wireless Comm.: Principles and Practice, second ed. Prentice-Hall PTR, 2002

[28] J. Robinson, K. Papagiannaki, C. Diot, X. Guo, and L. Krishnamurthy, "Experimenting with a Multi-Radio Mesh Networking Testbed," Proc. Fourth Int'l Workshop Wireless Network Measurements (WiNMee '05), Apr. 2005.

[29] J. So and N. Vaidya, "Multi-Channel MAC for Ad Hoc Networks: Handling Multi-Channel Hidden Terminals Using a Single Transceiver," Proc. ACM MobiHoc '04, May 2004

[30] E. Vergetis, E. Pierce, M. Blanco, and R. Guerin, "Packet-Level Diversity-From Theory to Practice: An 802.11-Based Experimental Investigation," Proc. ACM MobiCom '06, Sept. 2006.

[31] S.-L. Wu, C.-Y. Lin, Y.-C. Tseng, and J.-P. Sheu, "A New MultiChannel MAC Protocol with On-Demand Channel Assignment for Multi-Hop Mobile Ad Hoc Networks," Proc. Fifth Int'l Symp. Parallel Architectures, Algorithms and Networks (ISPAN '00), Dec. 2000.

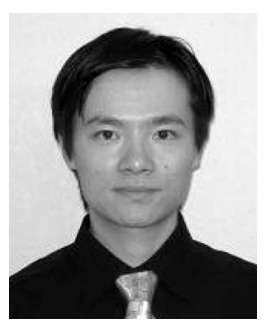

Pan $\mathbf{L i}$ received the $\mathrm{BE}$ degree in electrical engineering from Huazhong University of Science and Technology, Wuhan, China, in 2005, and the PhD degree in electrical and computer engineering from the University of Florida, Gainesville, in 2009, respectively. $\mathrm{He}$ is currently an assistant professor in the Department of Electrical and Computer Engineering, Mississippi State University. His research interests include capacity and connectivity analysis, medium access control, routing algorithms, and cross-layer protocol design in wireless networks. He is a member of the IEEE and the ACM.

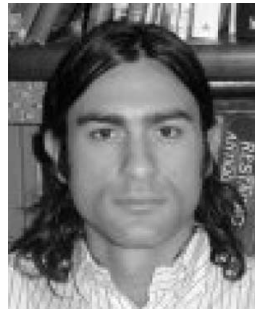

Nicola Scalabrino received laurea and master's degrees from the University of Pisa, Italy, both in 2004. During his $\mathrm{PhD}$ path at the University of Pisa, he cooperated with both the Institute of Informatics and Telematics (IIT), Italian National Research Council (CNR), and CREATE-NET, Trento. Currently, he is with Thales-I D3S, where he is lead engineer of transmission networks (i.e., fiber optic, IP, and SDH networks) for metro projects. His research interests focus on wireless networks with emphasis on MAC and routing protocols for IEEE 802.16 and IEEE 802.11 mesh networks.

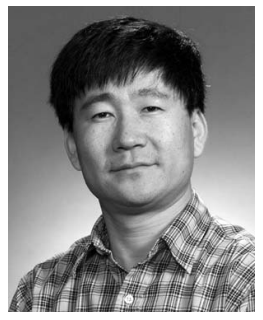

Yuguang (Michael) Fang received the $\mathrm{PhD}$ degree in systems engineering from Case Western Reserve University in January 1994 and the $\mathrm{PhD}$ degree in electrical engineering from Boston University in May 1997. He was an assistant professor in the Department of Electrical and Computer Engineering, New Jersey Institute of Technology, from July 1998 to May 2000. He then joined the Department of Electrical and Computer Engineering, University of Florida, Gainesville, in May 2000 as an assistant professor; he then got an early promotion to an associate professor with tenure in August 2003 and to a full professor in August 2005. He holds a University of Florida Research Foundation (UFRF) Professorship from 2006 to 2009. $\mathrm{He}$ has published more than 200 papers in refereed professional journals and conference proceedings. He is also active in professional activities. He has served on several editorial boards of technical journals including the IEEE Transactions on Communications, the IEEE Transactions on Wireless Communications, the IEEE Transactions on Mobile Computing, and the ACM Wireless Networks. He has been actively participating in professional conference organizations such as serving as the Steering Committee cochair for QShine, the Technical Program vice chair for IEEE INFOCOM 2005, Technical Program Symposium cochair for IEEE Globecom 2004, and a member of the Technical Program Committee for IEEE INFOCOM (1998, 2000, and 2003-2009). He received the US National Science Foundation Faculty Early Career Award in 2001 and the US Office of Naval Research Young Investigator Award in 2002. He is the recipient of the Best Paper Award in the IEEE International Conference on Network Protocols (ICNP) in 2006 and the recipient of the IEEE TCGN Best Paper Award in the IEEE High-Speed Networks Symposium at IEEE Globecom in 2002. He is a fellow of the IEEE and a member of the ACM. 


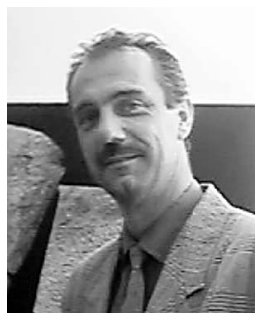

Enrico Gregori received the laurea degree in electronic engineering from the University of Pisa in 1980. In 1981, he joined the Italian National Research Council (CNR), where he is currently a CNR research director in the Institute of Informatics and Telematics. In 1986, he held a visiting position at the IBM research center in Zurich, working on network software engineering and on heterogeneous networking. He has contributed to several national and international projects on computer networking. He has authored more than 100 papers in the area of computer networks, published in international journals and conference proceedings, and is coauthor of the book Metropolitan Area Networks (Springer, 1997). He was the general chair of the IFIP TC6 conferences, Second International IFIP-TC6 Networking Conference (Networking 2002) and the Eighth International Conference Personal Wireless Communications (PWC 2003), and the Fourth Annual IEEE International Conference on Pervasive Computing and Communication (PERCOM 2006). He served as a guest editor for the Networking 2002 journal special issues of Performance Evaluation, Cluster Computing, and ACM/ Kluwer Wireless Networks. He is on the editorial boards of Cluster Computing, Computer Networks, and Wireless Networks. His current research interests include ad hoc networks, sensor networks, wireless LANs, quality of service in packet-switching networks, and evolution of TCP/IP protocols.

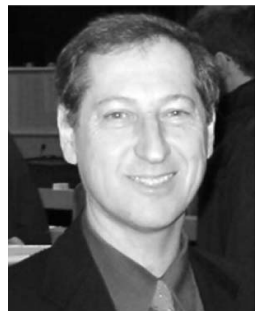

Imrich Chlamtac is the president of CREATENET, a nonprofit international research institute, and the Honorary Bruno Kessler Professor at the University of Trento, Italy. In the past, he was with Technion and the University of Massachusetts, Amherst, and DEC Research and helped found several successful technology firms, including Consip Ltd. and BCN Inc., one of the largest system integrators in Central Europe. He has held various chaired professorships in the US and Europe, including the Distinguished Chair in Telecommunications Professorship at the University of Texas at Dallas, Sackler Professorship at Tel Aviv University, "University Professorship" at the Budapest University of Technology and Economics, and Honorary Professorship from the Beijing University of Posts and Telecommunications. He is the recipient of numerous professional awards, including Fulbright Scholar, the ACM Award for Outstanding Contributions to Research on Mobility, and the IEEE Award for Outstanding Technical Contributions to Wireless Personal Communications. He published more than 400 refereed journal and conference articles and is listed among ISI's Highly Cited Researchers in Computer Science. He is a coauthor of several books, including the first book on local area networks (1980) and the Amazon.com bestseller Wireless and Mobile Network Architectures (John Wiley \& Sons). He has widely contributed to the scientific community as part of activities of the IEEE, the ACM, and SPIE and, more recently, as chair of ICST Scientific Council. He is also the founder and chair of ACM SIGMOBILE, the founder steering committee chair or chair of several leading conferences, including ACM MobiCom and ACM SIGCOMM and conferences cosponsored by ICST, Create-Net, and IEEE societies, including Broadnets, TRIDENTCOM, SecureComm, COMSWARE, WiOpt, MobiQuitous, and others. He serves as the founding editor in chief of ACM/Springer Wireless Networks and ACM/ Springer Journal on Special Topics in Mobile Networks and Applications. $\mathrm{He}$ is a fellow of the IEEE and the ACM.

$\triangleright$ For more information on this or any other computing topic, please visit our Digital Library at www.computer.org/publications/dlib. 